\title{
handicap et odontologie : propositions d'avenir
}

RÉSUMÉ En s'appuyant sur la loi 2005-102 du 11 février 2005, cet article vise à définir les notions de handicap, déficience et dépendance afin de déterminer la spécificité de la prise en charge de ces patients à travers la règle des trois «A» :

- accessibilité,

- adaptation,

- approche comportementale.

Après un panorama de l'état actuel de la prise en charge de ces patients, des propositions sont émises afin de donner des pistes de recherche sur l'amélioration significative

\section{ANASTASIO}

Département d'Odontologie, Responsable d'unité fonctionnelle, BP 60327

57126 Thionville Cedex.

\section{HEIN-HALBGEWACHS}

Département d'Odontologie du C.H.R. Metz-Thionville.

\section{DROZ}

Département d'Odontologie Pédiatrique,

Faculté d'Odontologie de Nancy.

\section{E. GERARD}

Département d'Odontologie du C.H.R. Metz-Thionville. de cette prise en charge au regard de la loi 2005-102 du 11 février 2005.

\section{MOTS CLÉS}

handicap

soins spécifiques

soins dentaires 


\section{introduction :}

\section{loi ${ }^{\circ}$ 2005-102 du 11 février 2005}

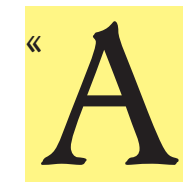

rt. 2. - Constitue un handicap, au sens de la présente loi, toute limitation d'activité ou restriction de participation à la vie en société subie dans son environnement par une personne en raison d'une altération substantielle, durable ou définitive d'une ou plusieurs fonctions physiques, sensorielles, mentales, cognitives ou psychiques, d'un polyhandicap ou d'un trouble de santé invalidant.»

«Toute personne handicapée a droit à la solidarité de l'ensemble de la collectivité nationale, qui lui garantit en vertu de cette obligation, l'accès aux droits fondamentaux reconnus à tous les citoyens ainsi que le plein exercice de sa citoyenneté.

L'état est garant de l'égalité de traitement des personnes handicapées sur l'ensemble du territoire et définit des objectifs d'actions.»

"À cette fin, l'action poursuivie vise à assurer l'accès de l'enfant, de l'adolescent ou de l'adulte handicapé aux institutions ouvertes à l'ensemble de la population et son maintien dans un cadre ordinaire de scolarité, de travail et de vie. Elle garantit l'accompagnement et le soutien des familles et des proches des personnes handicapées.» (Loi n 2005-102 du 11 février 2005).

Le dernier alinéa de cet article montre le degré d'implication de la société face à la personne handicapée par une volonté d'adaptation aux déficiences, incapacités et dépendances engendrées par le handicap dans le cadre ordinaire de vie au sein de notre société actuelle.

Cette loi devra donc logiquement s'appliquer dans un avenir proche à notre spécialité par une prise en charge spécifique.

La dépendance peut être définie comme étant l'incapacité à accomplir une tâche seul. En ce qui concerne l'odontologie, on peut reconnaître comme dépendante toute personne présentant une déficience fonctionnelle et pour laquelle les soins dentaires ne peuvent être réalisés de la même manière que pour les personnes de la population générale, en raison d'un obstacle médical.

Deux grands groupes peuvent être dégagés en fonction de l'âge de l'atteinte.

Le Groupe I désigne les atteintes de l'enfance (fig. 1 et 2 ) :

- les personnes autistes ;

- les personnes présentant une trisomie 21 ;

- les personnes présentant une infirmité d'origine cérébrale ;

- les personnes polyhandicapées ;

- les personnes présentant un retard psychomoteur ou un syndrome de déficience mentale ;

- les personnes présentant une déficience sensorielle. 


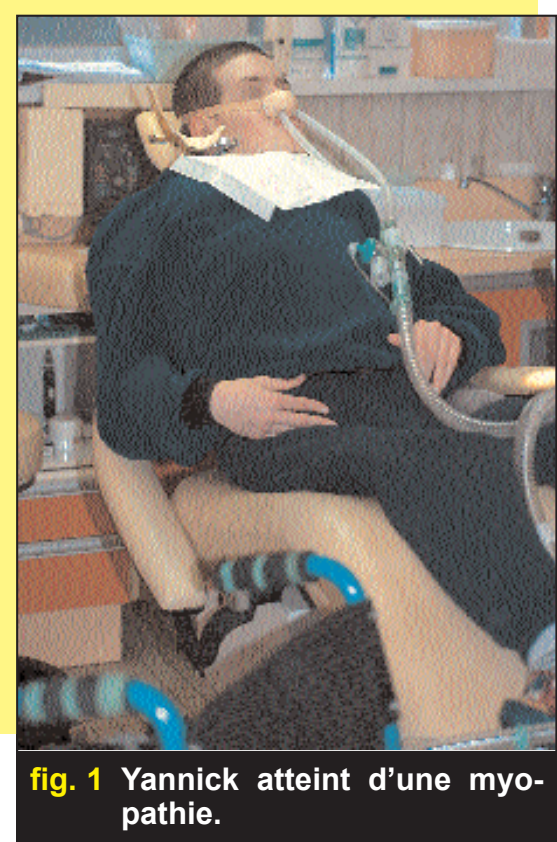

Le Groupe II désigne les atteintes de l'adulte (fig. 3 et $\mathbf{4}$ ) :

- les personnes atteintes de démence sénile ;

- les personnes présentant une maladie d'Alzheimer ;

- les personnes présentant une maladie dégénérescente évoluée : sclérose en

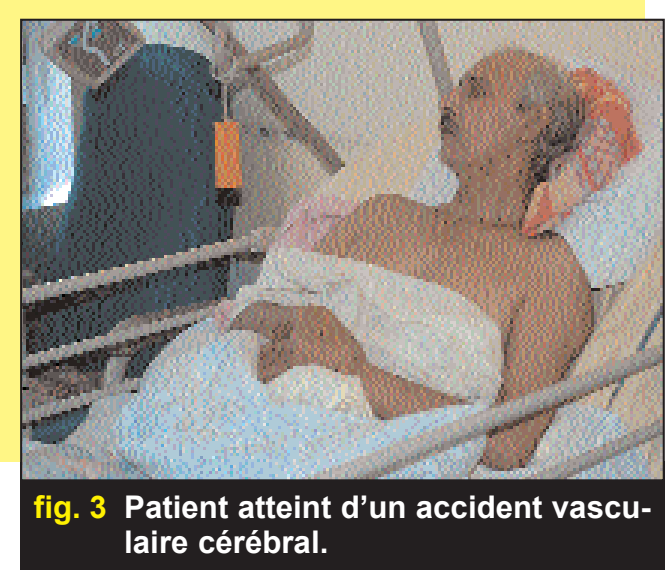

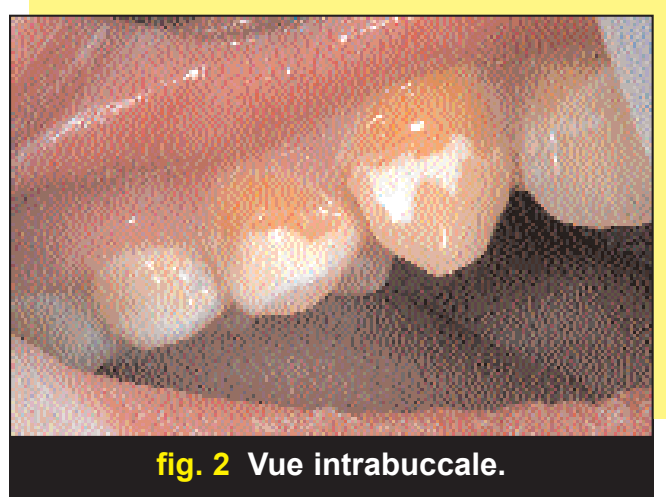

plaque, polyarthrite rhumatoïde, maladie de Parkinson, etc. ;

- les personnes présentant des séquelles cérébrales: encéphalopathies graves, accidents vasculaires cérébraux sévères, séquelles d'anévrismes, etc. ;

- les personnes âgées dépendantes.

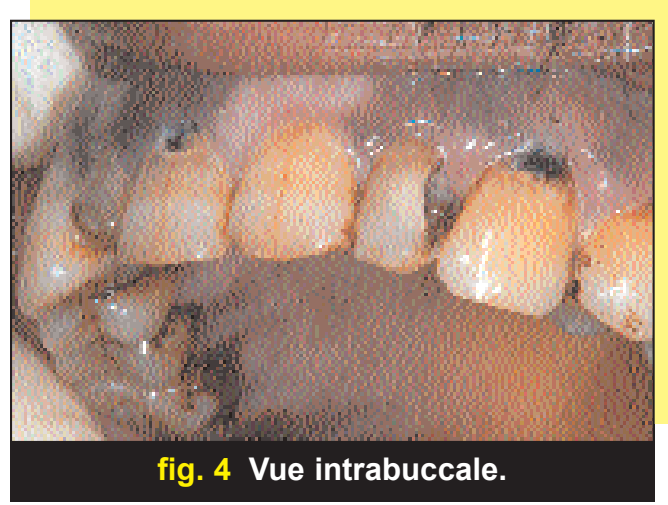

Actualités Odonto-Stomatologiques $n^{\circ} 239$, septembre 2007 


\section{la spécificité de la prise en charge}

Les soins dentaires sont identiques au reste de la population, pas leur réalisation. De plus, la pathologie dentaire rencontrée est accentuée par la déficience et la dépendance associées. Plus la déficience sera sévère, plus l'atteinte bucco-dentaire sera conséquente. Ces besoins sont donc spécifiques et liés au type de handicap rencontré.

La divergence de prise en charge des pathologies bucco-dentaires par rap-

- accessibilité

(fig. 5 à 7)

On peut définir l'accessibilité comme l'ensemble des moyens mis en œuvre pour permettre à un patient présentant une déficience d'accéder physiquement à ses besoins essentiels de vie.

«Les dispositions architecturales, les aménagements et équipements intérieurs et extérieurs des locaux d'habitation, qu'ils soient la propriété de per- port au reste de la population provient des difficultés rencontrées pour mettre en œuvre les thérapeutiques nécessaires.

Ces difficultés peuvent être résumées par la règle des trois «A»:

\section{Accessibilité \\ Adaptation \\ Approche comportementale.}

sonnes privées ou publiques, des établissements recevant du public, des installations ouvertes au public et des lieux de travail doivent être tels que ces locaux et installations soient accessibles à tous, et notamment aux personnes handicapées, quel que soit le type de handicap, notamment physique, sensoriel, cognitif, mental ou psy-

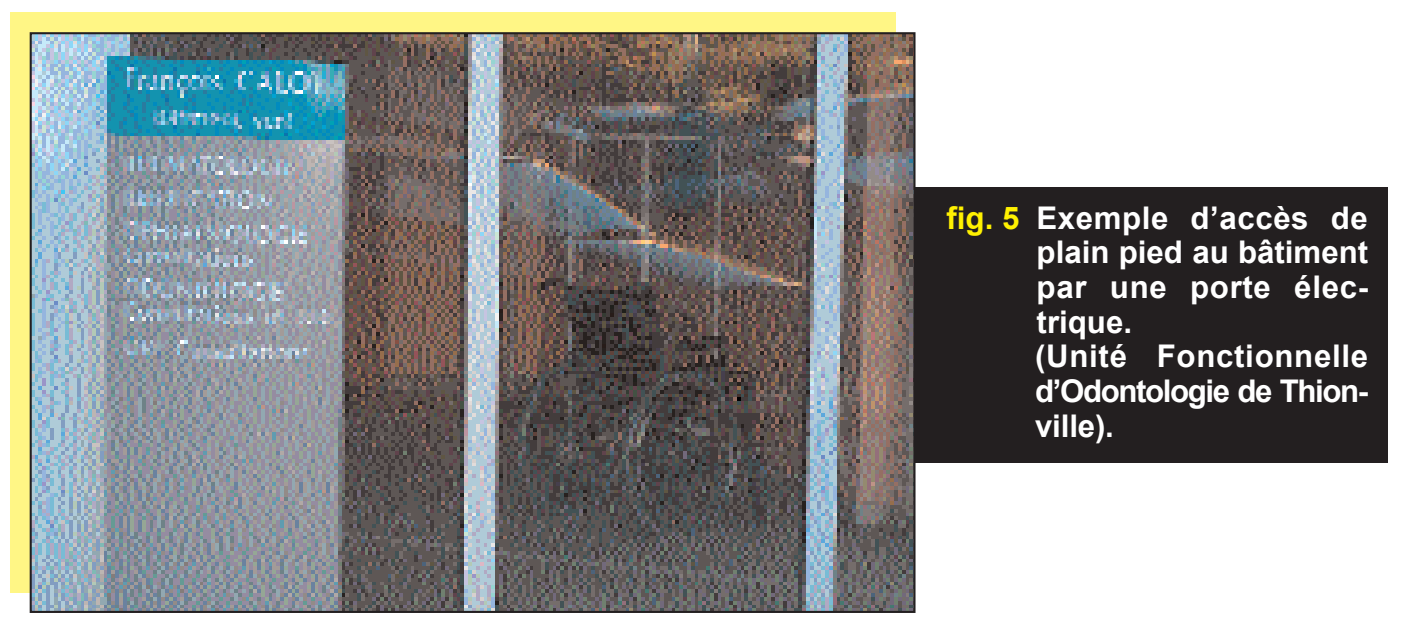




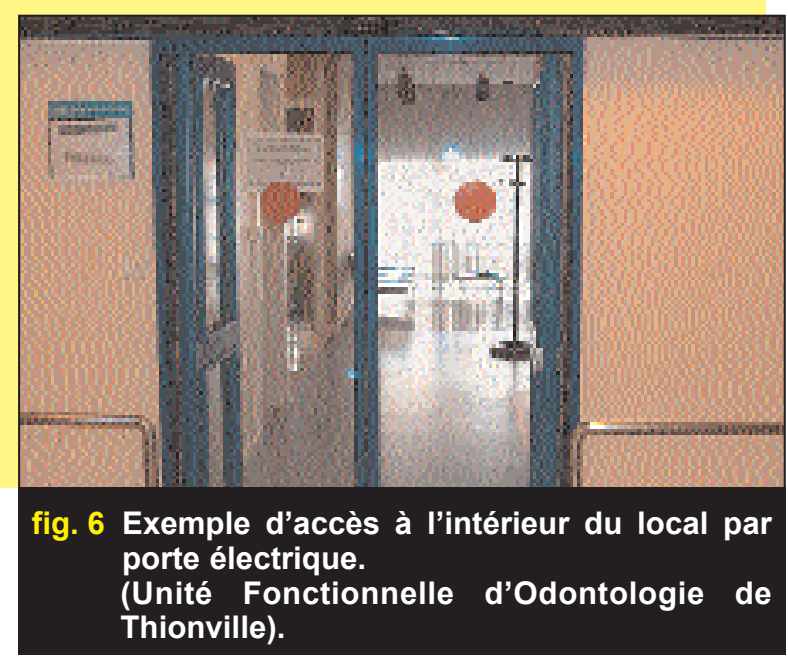

chique.» (Art 41, Loi $\mathrm{n}^{\circ}$ 2005-102 du 11 février 2005).

Les cabinets de soins bucco-dentaires doivent donc idéalement être accessibles aux personnes déficientes, que cette déficience soit d'ordre physique, sensorielle, cognitive, mentale ou psychique.

Cette accessibilité devra s'appliquer de l'entrée de l'immeuble jusqu'à l'intérieur du cabinet de soins.

- adaptation

(fig. 8)

On peut définir l'adaptation comme l'ensemble des moyens matériels mis en œuvre par l'équipe soignante pour permettre une prise en charge bucco-dentaire adaptée à la déficience de ces patients.

Particulièrement, les possibilités ergonomiques sont à étudier en mettant le patient déficient au centre de cette prise en charge.
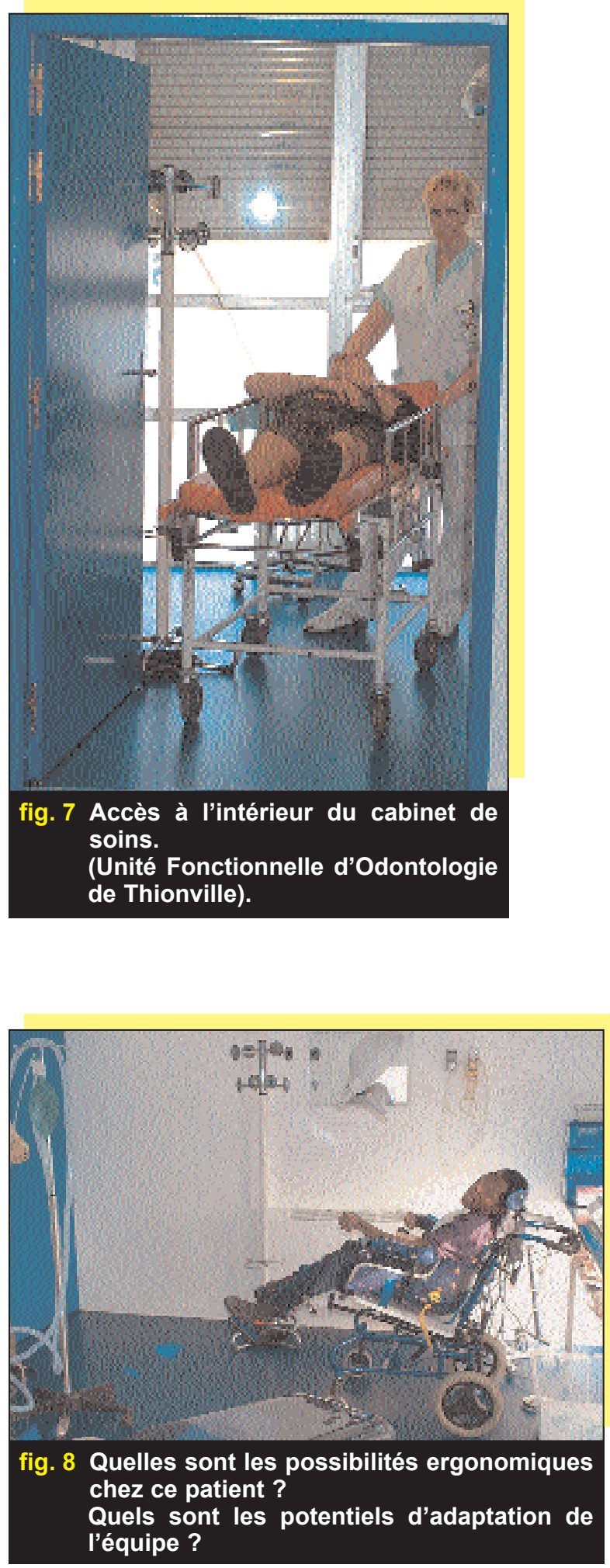
- approche comportementale (fig. 9)

On peut définir l'approche comportementale comme l'ensemble des possibilités de l'équipe soignante de prendre en charge un patient présentant une déficience cognitive, mentale ou psychique afin de donner une réponse adaptée à son comportement.

Cette approche est essentielle pour faciliter nos soins au cabinet.

\section{les données statistiques}

\section{ADULTES :}

Personnes âgées

dépendantes :

2300000

Noyau dur du handicap :

Déficiences mentales

et intellectuelles :

Maladies limitantes :

ENFANTS :

85000 bénéficieraient d'une allocation d'éducation spéciale.

220000 sont accueillis dans un établissement spécialisé.

650000

325000

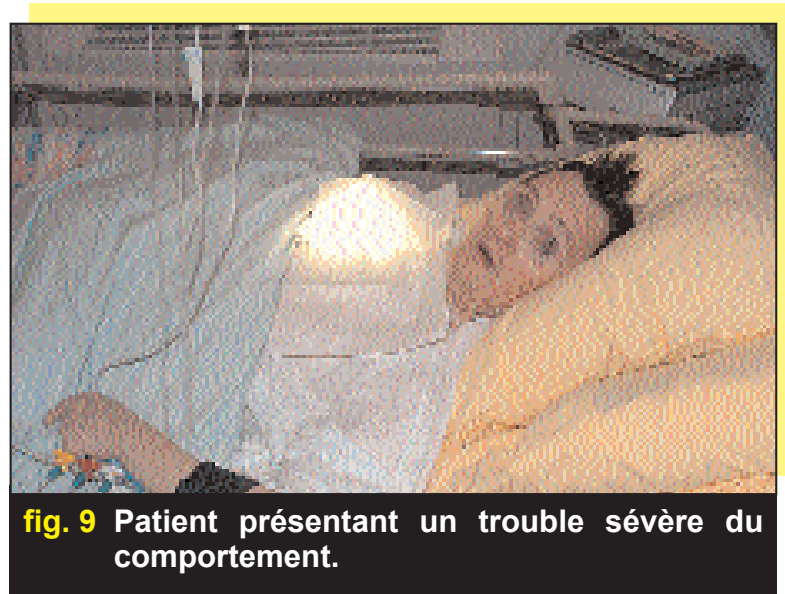

En réalité, il y aurait entre 1 et 5 millions d'individus selon la définition retenue $\mathrm{du}$ handicap et particulièrement lorsque l'on prend en compte les personnes souffrant d'une déficience grave ou d'un handicap au sens de désavantage.

L'accroissement de l'espérance de vie dans notre pays d'une part et, d'autre part, le vieillissement de la population va faire croître la fréquence des prises en charge. À l'horizon 2050, un personne sur trois sera âgée de 60 ans ou plus. Il s'agit bien d'un véritable défi à relever pour les années à venir.

\section{état actuel de la prise en charge}

La prise en charge de ces populations n'est pas structurée.

Absence d'organisation, de coordination et d'information.

Vide «bucco-dentaire» autour des personnes handicapées et âgées dépendantes.
Qui les soigne? Quels sont les moyens mis en œuvre?

Ce constat repose sur l'absence d'informations objectives au niveau national. Des prises en charge doivent exister à un niveau individuel, mais ces dernières se trouvent isolées. 
Les objectifs idéaux de cette prise en charge sont de trois ordres :

1. Le traitement afin de détecter et traiter les pathologies bucco-dentaires en prenant en compte la nature de la dépendance et des pathologies générales associées à cette dernière ainsi que l'évolution des problèmes buccodentaires liés à la maladie. Les plans de traitement sont définis en fonction de chaque patient en incluant les possibilités thérapeutiques par rapport aux difficultés spécifiques rencontrées lors des soins.

Ces possibilités thérapeutiques varient selon le degré de coopération du patient et de l'état bucco-dentaire initial.

- Certains patients seront traités sous anesthésie générale ou sédation intraveineuse (ambulatoire de préférence) si leur état général le permet.

- D'autres seront pris en charge sous sédation par inhalation (mélange équimolaire d'oxygène et de protoxyde d'azote : MEOPA).

La sédation consciente par inhalation de MEOPA étant un moyen sûr et efficace d'obtenir une anxiolyse et une analgésie légère dans le cadre de soins dentaires spécifiques.
- Les plus coopérants sont traités à l'état vigile au fauteuil en incluant une approche spécifique adaptée à chaque patient, qu'elle soit d'ordre psychologique, comportemental ou ergonomique.

2. La prévention par la mise en place d'un enseignement des méthodes de prévention de l'apparition des pathologies bucco-dentaires que sont la carie dentaire et les maladies parodontales. Cette prévention s'exerce $\mathrm{au}$ niveau du patient mais aussi et surtout au niveau des auxiliaires de vie et $d u$ milieu familial en tenant compte de la sévérité de la dépendance (elle sera d'autant plus efficace que la dépendance sera limitée).

3. Mise en place de conventions avec les différents établissements spécifiques que sont :

- les maisons d'accueil spécialisées ;

- les instituts médicaux éducatifs ;

- les foyers de vie ;

- les hôpitaux médico-gériatriques ; afin d'effectuer des visites annuelles de dépistage bucco-dentaire auprès de chaque pensionnaire (fig. 10).

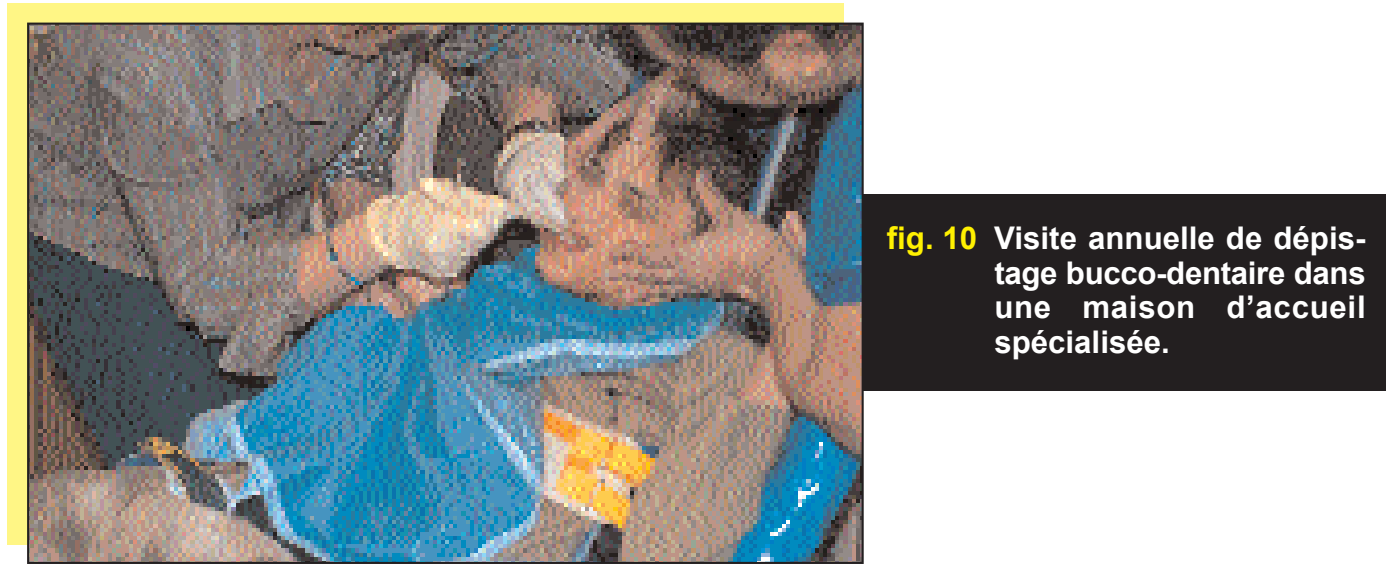




\section{les propositions}

- proposition $\mathrm{n}^{\circ} 1$

Recensement des praticiens prenant en charge ces patients :

- la création de réseaux permettra de connaître ces praticiens, l'accessibilité des cabinets dentaires et les possibilités de cette prise en charge. Ces réseaux devront inclure étroitement les praticiens libéraux et hospitaliers afin de tisser à un niveau régional une toile de praticiens complémentaires.

\section{- proposition $\mathrm{n}^{\circ} 2$}

Définition d'un cabinet dentaire ergonomique permettant de prendre en charge l'ensemble des patients handicapés ou dépendants.

Ce local devra être accessible à un lit de $110 \mathrm{~cm}$ de large, le patient devenant le centre de cette prise en charge et l'équipe soignante pouvant s'adapter à la déficience et aux conséquences du handicap (fig. 11).

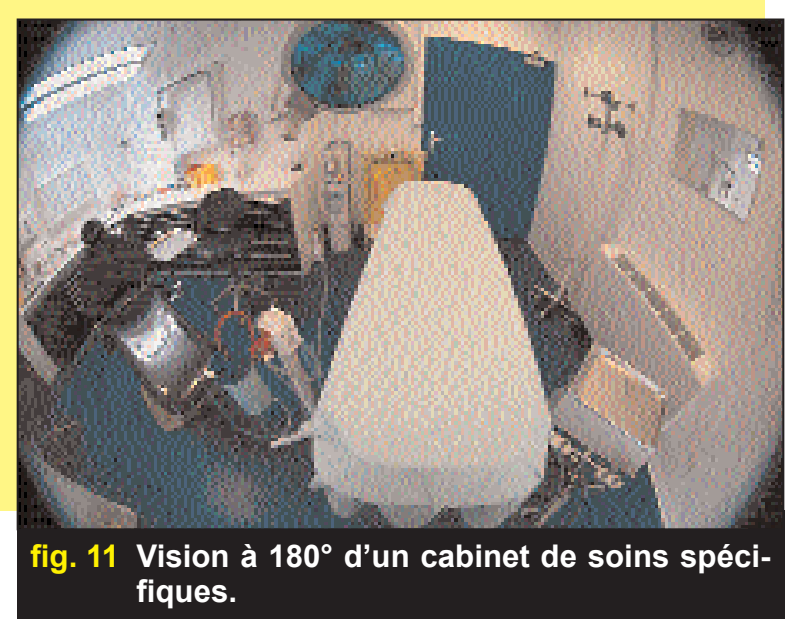

Cette configuration pourrait inspirer toute création d'un cabinet dentaire accessible, dans les années à venir, afin de faire face à ce problème et répondre aux impératifs de la loi du 11 février 2005.

\section{proposition $\mathrm{n}^{\circ} 3$}

Mesures compensatoires intégrant l'acte technique classique d'une part et d'autre part, les moyens supplémentaires mis en œuvre pour traiter le patient.

Ces mesures compensatoires pourront être par exemple un «forfait handicap» variable en fonction du type de handicap rencontré.

Elles permettront une rémunération des praticiens à la hauteur des moyens techniques et humains mis en œuvre. De plus, elles engendreront une proportion plus importante de praticiens à traiter ces patients dans leur cabinet.

En effet, la prise en charge de ces patients monopolise des moyens spécifiques et demande au praticien une disponibilité de temps bien supérieure par rapport à un sujet sain à acte identique réalisé.

- proposition $\mathrm{n}^{\circ} 4$

Création d'Unités Fonctionnelles pour Soins Spécifiques (U.F.S.S.).

Ces U.F.S.S. permettront la prise en charge de patients présentant des déficiences ou des dépendances lourdes.

Elle exigent des moyens techniques et humains indispensables : 
- locaux accessibles (parking, accès ambulances, porte d'accès large du bâtiment, ascenseur...) ;

- locaux adaptés avec une possibilité de prise en charge de tout type de situations cliniques (lit, brancard, chaise, coque moulée...) ;

- personnel paramédical formé ou ayant une expérience.

Chaque département devrait être pourvu d'au moins une U.F.S.S. afin de répondre à une nécessité de plus en plus urgente. En effet, l'accroissement prévisible des besoins en soins des personnes dépendantes dont le nombre ne cesse d'augmenter du fait d'une espérance de vie devenue plus grande que par le passé, ne peut que renforcer ce besoin.

Ces U.F.S.S. pourront être mises à disposition des praticiens libéraux qui le désirent afin de traiter eux-mêmes leurs patients, ceci dans un cadre juridique à définir.

Il semble évident que les structures hospitalières sont naturellement les mieux adaptées à la création de ces Unités Fonctionnelles de Soins Spécifiques. Des moyens devront être développés par l'Agence Régionale de l'Hospitalisation pour réaliser ces structures (ou les renforcer si elles existent) au sein des services hospitaliers d'Odontologie.

\section{- proposition $\mathrm{n}^{\circ} 5$}

Développement de la technique de sédation par inhalation de M.E.O.P.A. (Mélange Équimolaire d'Oxygène et de Protoxyde d'Azote) pour la prise en charge de patients présentant des troubles comportementaux (fig. 12).

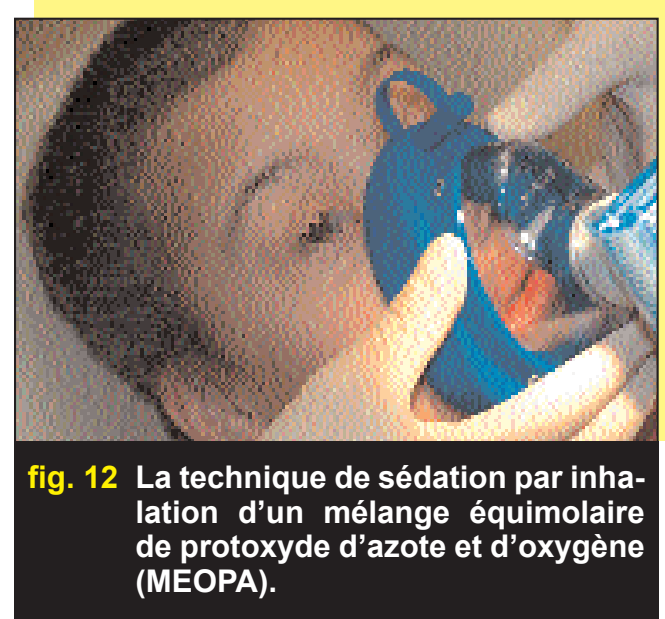

Les praticiens devront être obligatoirement formés à cette technique par la validation d'un diplôme universitaire.

Il est souhaitable que cette technique puisse être étendue aux praticiens libéraux ; l'A.M.M. de ce gaz médical étant réservée actuellement à l'exercice odontologique hospitalier.

De même, des mesures compensatoires devront être accordées afin de «compenser» le coût financier engendré par cette technique d'une part et d'autre part, le temps passé pour la mettre en œuvre.

\section{proposition $n^{\circ} 6$}

Formation des praticiens à la prise en charge des patients handicapés et dépendants afin de connaître les spécificités de chaque déficience ainsi que les pathologies associées, qu'elles soient bucco-dentaires ou médicales.

Ces formations théoriques seront complétées par des visites en institutions spécialisées pour prendre conscience des problèmes spécifiques rencontrés. 
De même, ces formations pourront inclure des stages hospitaliers au sein de centres odontologiques prenant en charge cette population spécifique.

Formation également des étudiants dans le cadre de leur cursus universitaire.

\section{- proposition $\mathrm{n}^{0} 7$}

Mise en place, dans chaque établissement accueillant des patients déficients ou dépendants lourds, d'une visite annuelle de dépistage de pathologies bucco-dentaires associée à une sensibi- lisation du personnel d'encadrement à la mise en place d'une hygiène dentaire adaptée.

Ces visites pourront être coordonnées par des organismes professionnels dans chaque département.

Elles devront être rendues obligatoires par les instances administratives de tutelle avec l'obligation d'une compensation financière au bénéfice du praticien ayant réalisé la visite.

Cette compensation sera forfaitaire et proportionnelle à la taille de l'établissement.

\section{conclusion}

La loi du 11 février 2005 est un véritable défi pour notre société face à l'intégration de la personne handicapée dans notre cadre de vie. Il s'agit d'un projet ambitieux qui mettra très certainement des années à s'installer au quotidien.

Plusieurs pays, comme les pays scandinaves, ont pris une avance considérable dans la place de la personne handicapée dans la société. Ces sociétés ont su mettre l'être humain au centre de leur modèle de vie en y intégrant la personne handicapée (fig. 13).
Notre profession devra, dans un avenir proche, se déterminer face à cette loi, certes exigeante, mais cependant tellement nécessaire. L'objectif semble difficile à atteindre tant les obstacles à franchir sont nombreux et immenses.

Les propositions émises ne sont que diverses pistes à explorer afin de réaliser cet objectif éthique, ambitieux dans sa dimension humaine, qu'est la prise en charge de la personne handicapée dans nos cabinets.

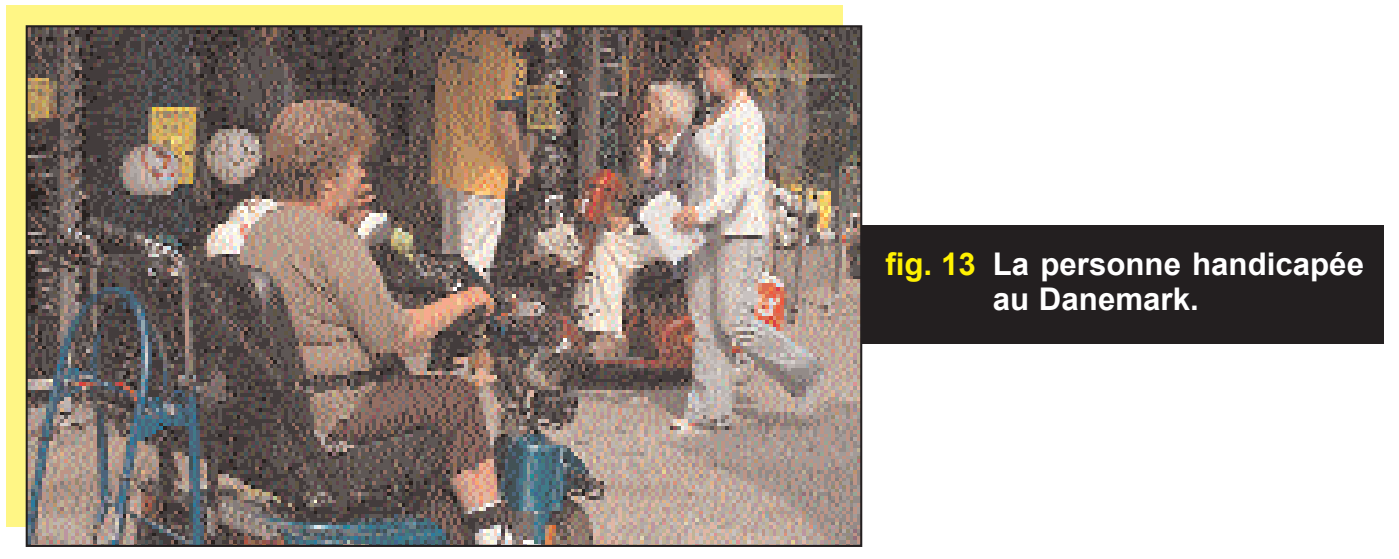




\section{ouvrages à consulter}

Loi $n^{\circ}$ 2005-12

du 11 février 2005

pour l'égalité des droits et

des chances, la participation

et la citoyenneté

des personnes handicapées.

Triomphe A, Alix MP.

Les données statistiques

relatives aux personnes

handicapées.

Actualité et dossier

en santé publique,

$n^{\circ} 6$ mars 1994.

Goillot C, Mormiche P.

Enquête

«handicaps,

incapacités, dépendances»

en institution en 1998, INSEE.

Résultats, collection

Démographie-Société,

n 83-84, 2001.

Ville I, Ravaud JF, Letourmy A. Les désignations du handicap, des incapacités déclarées à la reconnaissance administrative. Revue Française des Affaires Sociales,

n 1-2, janvier-juin 2003.

Le Bouler S.

Perspectives des besoins

$d$ 'hébergement

en établissement pour

les personnes âgées

dépendantes.

Rapport de la mission

du Commissariat Général

du Plan, juillet 2005.

Brouard C.

Le handicap en chiffres.

Centre Technique National

d'Études et de Recherches

sur les Handicaps et les

Inadaptations. Février 2004.

Bochelen A.

La prise en charge

bucco-dentaire

de la personne âgée

dépendante.

Thèse Chir. Dent. Nancy 1 , 2003.

Hennequin $\mathrm{M}$, Tubert $\mathrm{S}$.

Prise en charge par les chirurgiens-dentistes du Puy-de-Dôme

des personnes

handicapées.

Inf Dent 1999;81(38):2861-2878.

Anastasio D, Giraud E.

Les soins dentaires

chez le patient handicapé

par diazanalgésie vigile

ambulatoire.

Spécial Anesthésie.

Actual Odontostomatol

1992:294-298.

Brutel C, Omalek L.

Projections démographiques

pour la France, ses régions

et ses départements

(horizon 2030-2050).

INSEE, Résultats Société n 16 , juillet 2003.

Gérard E.

SROSS Odontologie,

région Lorraine.

Agence Régionale

de l'Hospitalisation.

Nancy, 2006.

\section{Disability and dental care: suggestions in future}

D. ANASTASIO, L. HEIN-HALBGEWACHS, D. DROZ, E. GERARD

This article defines the notion of disability, deficiency and dependence and determines the special needs of this patients during dental treatment.

Our guidelines are based on three rules:

- accessibility
- adaptation

- behavioural approach.

Suggestions are described for the improvement of the dental treatment of these patients according to theirs special needs in France.

keywords: disability, special needs, dental treatment. 